\title{
Random sequential adsorption of unoriented rectangles onto a plane
}

\author{
R. Dennis Vigil and Robert M. Ziff \\ Department of Chemical Engineering, The University of Michigan, Ann Arbor, Michigan 48109
}

(Received 1 March 1989; accepted 12 May 1989)

\begin{abstract}
Random sequential adsorption of nonoverlapping rectangles of arbitrary orientation onto a continuous plane was investigated by computer simulation. The approach to the jamming limit was found to obey Feder's law for a wide range of rectangle aspect ratios. The coverage fraction at the jamming limit was found to depend upon the aspect ratio of the adsorbed rectangles, with a maximum in the jamming coverage occurring at aspect ratios $\approx 2$.
\end{abstract}

Random sequential adsorption (RSA) is an irreversible process in which objects are placed, one at a time, randomly in space and with random orientation subject to the condition that they do not overlap previously adsorbed objects. Eventually no more objects can be adsorbed, and the jamming limit is reached. This process is of interest in a variety of fields including statistical, ${ }^{1,2}$ chemical, ${ }^{3,4}$ and biological ${ }^{5,6}$ physics. The one-dimensional problem, sometimes called the carparking problem, was first solved by Flory ${ }^{3}$ for discrete space, in connection with the irreversible binding of ligands on polymer chains. Since Flory's early work, several analytical solutions for the maximum coverage and the distribution of gaps at the jamming limit for both the discrete and continuous one-dimensional cases have been found. ${ }^{7-10}$

In contrast to the one-dimensional case, no analytical solutions for the coverage at the jamming limit have been obtained for higher dimensional problems. Indeed, Kendall and Moran ${ }^{11}$ have argued that the prospects of obtaining such solutions are remote. Consequently, RSA in two and higher dimensions has been studied mainly with the aid of computer simulations. ${ }^{5-6,12-20}$ Feder $^{5}$ investigated the kinetics of RSA of disks on a plane and postulated the following scaling law for the long-time behavior of the coverage fraction, $\theta$,

$$
\theta_{j}-\theta(\tau) \sim \tau^{-1 / d}, \quad \tau \rightarrow \infty,
$$

where $\tau$ is the cumulative number of attempts to adsorb objects (both successful and not), $d$ is the dimensionality of the system, and $\theta_{j}$ is the coverage at the jammed state. Pomeau ${ }^{\prime}$ and Swendsen ${ }^{2}$ have constructed arguments based upon geometrical probability that confirm this scaling law for RSA of disks on a plane and Swendsen ${ }^{2}$ has argued that it may be generalized for arbitrary shapes, provided that objects are adsorbed with random orientations. Other models of RSA, however, do not necessarily obey Eq. (1). For example, Swendsen ${ }^{2}$ has shown that the kinetics of RSA of aligned squares does not follow Eq. (1). Likewise, Barker and Grim$\operatorname{son}^{19}$ found Eq. (1) is violated for RSA of binary mixtures of various shapes on a square lattice. Rosen et al. ${ }^{17}$ examined the RSA of spherical particles onto small spherical substrates, and their results showed that Feder's law is not obeyed when the relative sizes of the adsorbing spheres to the substrate spheres is greater than a critical value. Most recently, Schaaf and Talbot ${ }^{20}$ have given an expression for the kinetics of RSA of disks on a plane at short times, before the onset of the asymptotic behavior given by Eq. (1) is reached.

In this paper, we present the results of a computer simulation of the random sequential adsorption of rectangles with arbitrary orientation upon a continuous plane. Such a study is of interest in connection with processes such as the irreversible adsorption of long, rodlike polymers upon a substrate. ${ }^{21}$ In particular, we are interested in how the jamming coverage is related to the aspect ratio of the adsorbing objects.

All simulations were performed on an Apollo Domain 4000 computer. Centerpoints and angular orientations of rectangles of a given aspect ratio were generated randomly with uniform probability for a square target area with sides of unit length. Aspect ratios were varied from 1 to 64 where in all cases the ratio of the rectangle area to the target area was $<0.0004$. Periodic boundary conditions were used in order to minimize boundary effects. In all, approximately 20 weeks of computer time were used in this work.

In order to determine if the adsorption of a trial rectangle would result in an overlap with a previously adsorbed rectangle, the following algorithm was used. First, the distance, $D_{i}$, between the centerpoints of the trial rectangle and

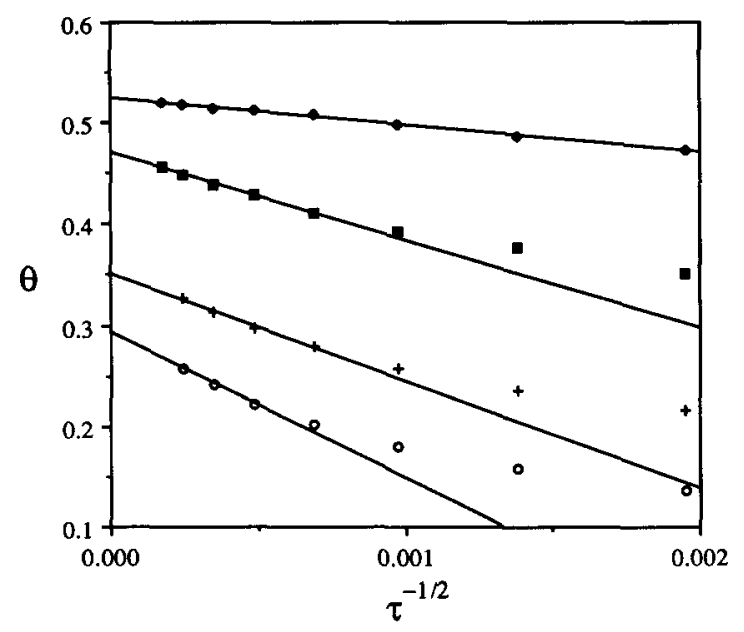

FIG. 1. Surface coverage $\theta$ as a function of $\tau^{-1 / 2}$, where $\tau$ is the total number of adsorption attempts. Representative simulations are shown for various aspect ratios, $(\Delta) \alpha=1$, (D) $\alpha=8,(+) \alpha=32$, (O) $\alpha=64$. The lines show that Feder's law is approached at long times. 
the $i$ th adsorbed rectangle was determined. If $D_{i}>\left(w^{2}\right.$ $\left.+l^{2}\right)^{1 / 2}$ where $w$ and $l$ are the width and length of the adsorbing rectangles, then we concluded that no overlap could occur between the trial rectangle and the $i$ th rectangle. Likewise, if $D_{i} \leqslant w$, then the trial rectangle must have overlapped the $i$ th rectangle and this attempt was rejected. For cases in which $w<D_{i} \leqslant\left(w^{2}+l^{2}\right)^{1 / 2}$, each of the four line segments forming the perimeter of the trial rectangle was checked for intersection with the four line segments forming the perimeter of the $i$ th adsorbed rectangle. Simulations were run up to $2^{27}$ attempted adsorption events and 3-5 simulations were carried out at each aspect ratio of $1,1.125,1.25,1.5,2,2.5,3$, $4,8,16,32,64$, and 256.

The surface coverage was calculated as a function of $\tau$ by the following relation:

$$
\theta(\tau)=\frac{N(\tau) l w}{L^{2}}
$$

where $N(\tau)$ is the number of successfully adsorbed rectangles at trial $\tau$ and $L$ is the length of a side of the target square ( $L=1$ in this study). The coverage, $\theta$, is plotted against $\tau^{-1 / 2}$ for various aspect ratios in Fig. 1. The plots in all cases become linear at long times, suggesting that Feder's scaling
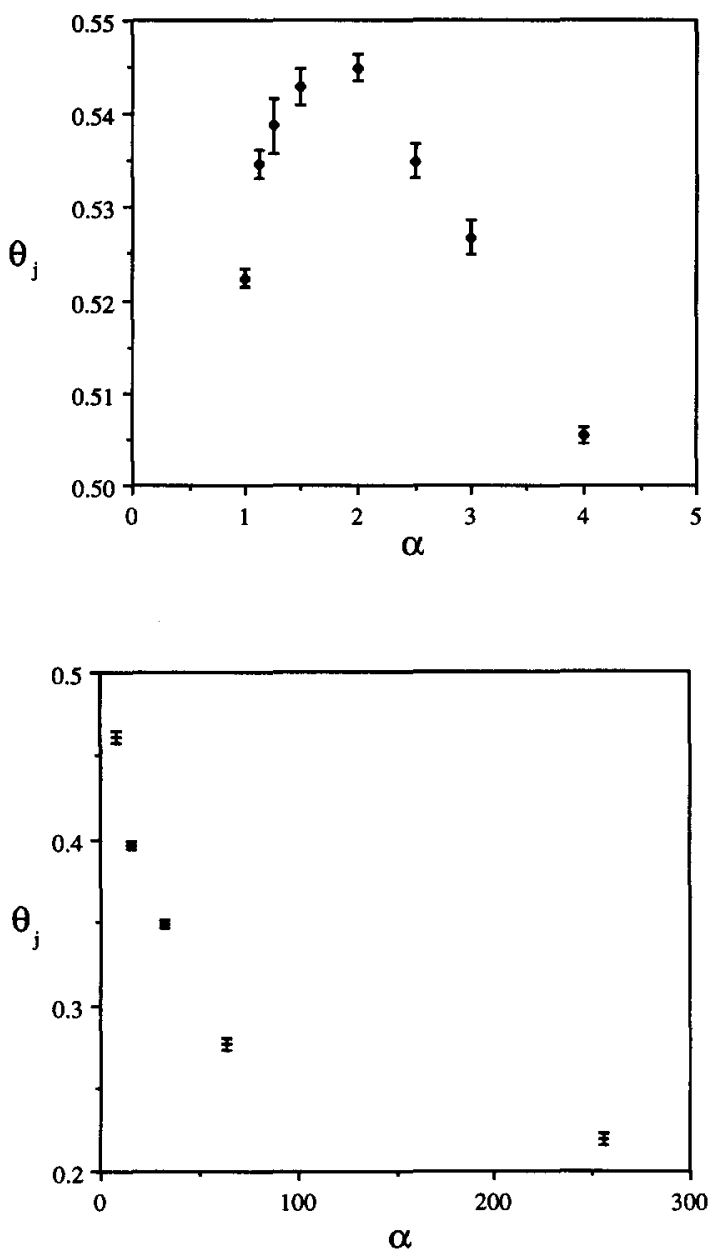

FIG. 2. Jamming coverage, $\theta_{j}$, vs aspect ratio, $\alpha$. (a) $\alpha$ between 1 and 4 and (b) $\alpha$ between 8 and 256. Error bars represent 1 standard deviation from the mean, which was obtained from 3-5 simulations. law is satisfied for randomly oriented rectangles, and thus confirming Swendsen's ${ }^{2}$ analysis. However, we note that Fig. 1 suggests that as $\alpha$ increases, the time necessary to reach Feder's scaling regime also increases, implying that the scaling Eq. (1) may be valid only for $\tau \gg f(\alpha)$, where $f(\alpha)$ is some monotonically increasing function of $\alpha$. An estimate of the coverage at the jammed state, $\theta_{j}$, may be found by extrapolating the long-time data to $\tau=\infty$ using Eq. (1). These $\theta_{j}$ are plotted as a function of the aspect ratio, $\alpha=l / w$, in Figs. 2(a) and 2(b).

Notice that Fig. 2(a) shows a distinct peak in the jammed coverage at aspect ratios $\approx 2$ which then falls off at higher aspect ratios. Although the existence of a peak may seem surprising, it may be understood from the following observations. In the early stages of RSA, relatively few overlaps occur and almost every trial rectangle is adsorbed. Therefore, the orientations of these adsorbed rectangles are not strongly correlated with other neighboring rectangles.
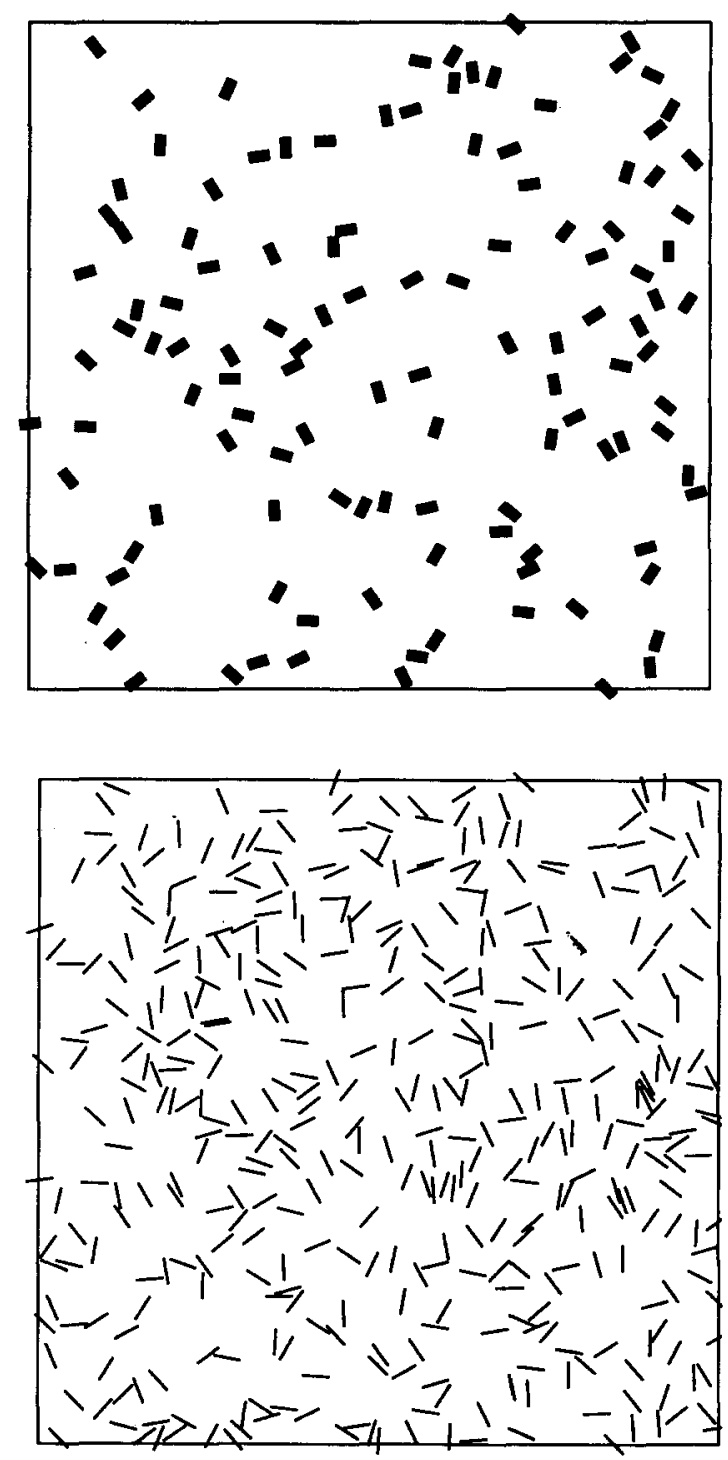

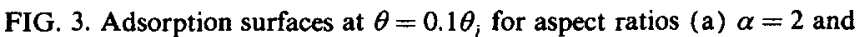
(b) $\alpha=16$. Because the image is a discretized representation of the surface, some rectangles may appear to have irregular shapes or may seem to be touching other rectangles. 
Thus, during the first stages of RSA we expect that relatively large areas of target surface will be used inefficiently due to the highly random packing configurations. A measure of how this short-time "wasting" effect depends upon the aspect ratio may be found from the following consideration. The packing efficiency at short times will depend upon how uniformly the rectangles fill space, which is given by the ratio of the actual area covered by a rectangle to the area swept out by rotating the rectangle through all possible orientations. Thus, we define packing efficiency, $\eta$, by

$$
\eta(\alpha) \equiv \frac{w l}{\pi\left(w^{2}+l^{2}\right) / 4}=\frac{4 \alpha}{\pi\left(\alpha^{2}+1\right)} .
$$

Thus $\eta \sim 1 / \alpha$ as $\alpha$ becomes large, implying that this wasting effect at short times will be more pronounced for rectangles with high aspect ratios than for those with low aspect ratios. Visual verification of this effect may be seen by comparing Figs. 3(a) and 3(b), which show adsorption surfaces at $\theta \approx 0.1 \theta_{j}$ for rectangles with $\alpha=2$ and 16 , respectively.

At long times, however, an ordering effect occurs that
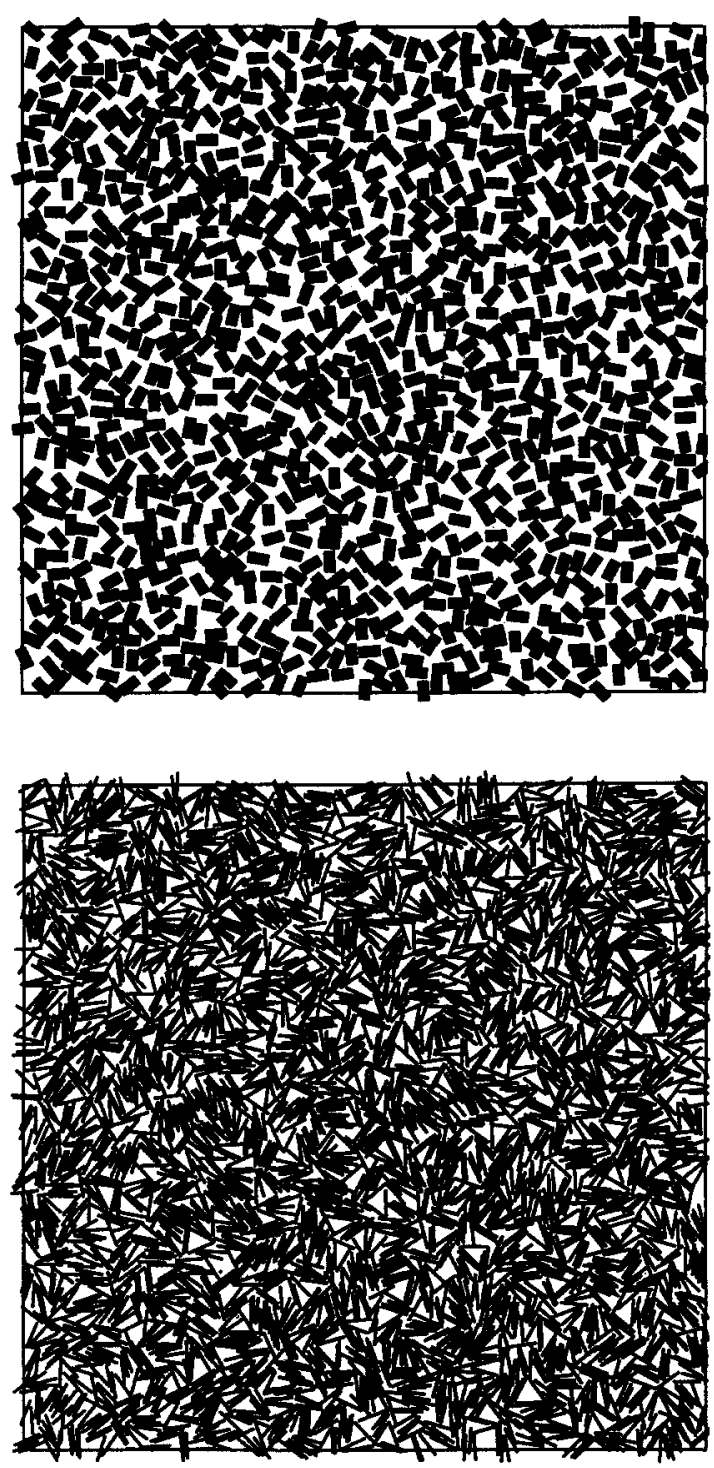

FIG. 4. Adsorption surfaces at $\theta=0.9 \theta_{j}$ for aspect ratios (a) $\alpha=2$ and (b) $\alpha=16$ becomes more pronounced as $\alpha$ increases. At high coverages, many overlaps occur and few trial rectangles are able to successfully adsorb. Trial rectangles with an angular orientation similar to the orientations of its previously adsorbed neighbors have a smaller chance of overlapping with these neighbors and thus have a greater chance of successfully adsorbing than do trial rectangles with angular orientations very different than those of its neighboring rectangles. As a result, angular orientations of subsequently adsorbing rectangles become more correlated with neighboring rectangles and the packing becomes more ordered and efficient as $\theta$ increases. This long-time ordering effect would be expected to become more pronounced as $\alpha$ increases, since rectangles with high aspect ratios must have almost identical angular orientations with its nearest neighbors in order to successfully adsorb at these high coverages. Figures 4(a) and 4(b) show adsorption surfaces for $\alpha=2$ and 16 at a coverage $\theta \approx 0.9 \theta_{j}$. Short-distance correlations in the angular orientations of rectangles are more evident in Fig. 4(b).

In summary, at short times a low value of $\alpha$ would be expected to lead to more efficient packing while at long times a high value of $\alpha$ is more efficient. The combined effect evidently produces a maximum in $\theta_{j}(\alpha)$ at some value of $\alpha$. We
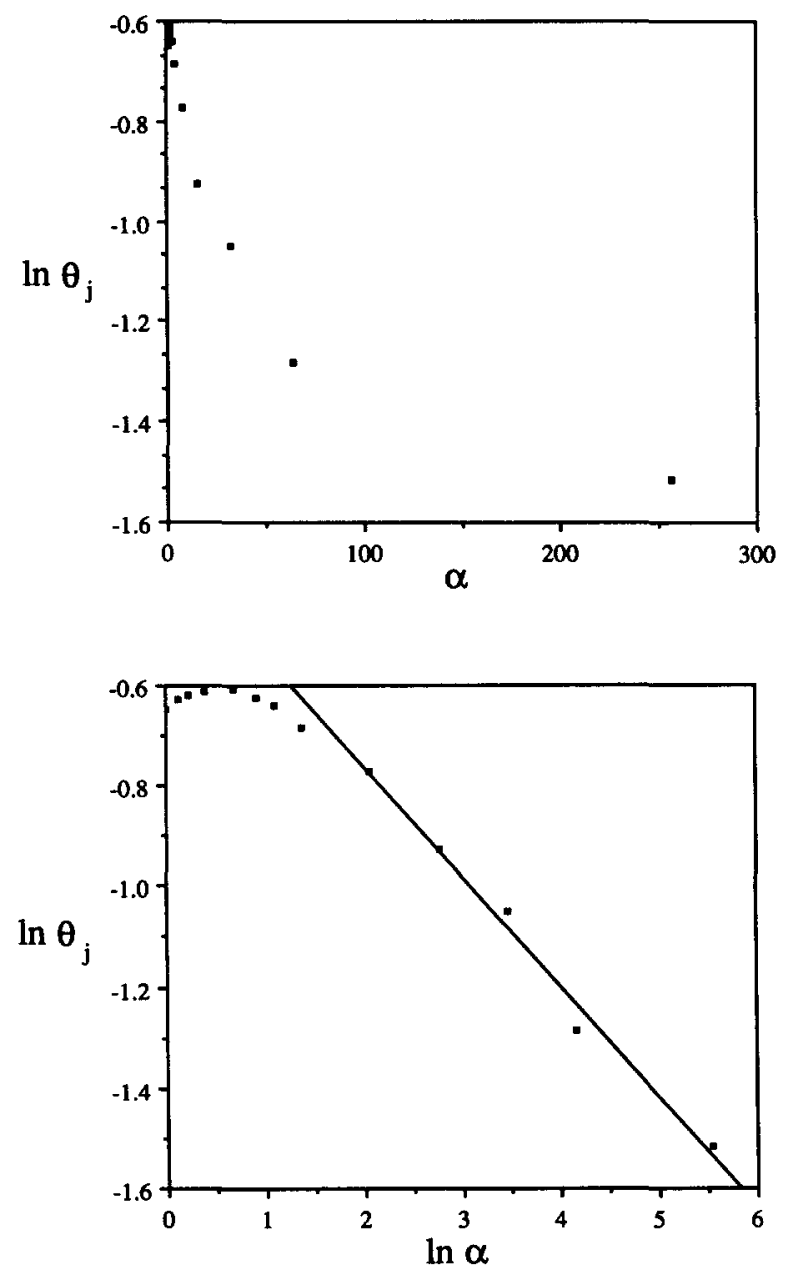

FIG. 5. Log-normal (a) and $\log -\log (\mathrm{b})$ plots of $\theta_{j}$ vs $\alpha$. The slope of the line in (b) is -0.22 . 
have found $\alpha_{\max } \approx 2$, corresponding to $\theta_{j, \max } \approx 0.545$, which is about $4 \%$ higher than the coverage for unoriented squares, $\theta_{j}(1) \approx 0.523$. In comparison, Feder ${ }^{5}$ reports $\theta_{j}=0.562$ for aligned squares and $\theta_{j}=0.547$ for circles.

Lastly, we consider the asymptotic behavior of $\theta_{j}(\alpha)$ as $\alpha \rightarrow \infty$. We expect that $\theta_{j} \rightarrow 0$ as $\alpha \rightarrow \infty$ and thus conjecture the simple forms $\theta_{j} \sim e^{-a \alpha}$ or $\theta_{j} \sim \alpha^{-b}$ where $a$ and $b$ are constants. Log-normal and log-log plots of our data shown in Figs. 5(a) and 5(b) clearly indicate that the data is more consistent with the power-law form with $\theta_{j} \approx 0.73 \alpha^{-0.22}$.

Note added in proof. We have just received a preprint by Talbot, Tarjus, and Schaaf, ${ }^{22}$ in which the RSA of randomly oriented ellipses is studied. They argue, because of orientational restrictions, that the long time behavior should be $\theta_{j}$ $-\theta(\tau) \sim a \tau^{-p}$ with $p=1 / 3$ rather than $p=1 / 2$ of Feder's law. Their arguments apply to squares and rectangles as well as ellipses. We note that their results are in conflict with those of Swendsen, who argues that Feder's law applies to all randomly oriented objects. We have reexamined our data by making a plot of $\ln [\theta(2 \tau)-\theta(\tau)]$ vs $\ln \tau$, which will give a straight line with slope $-p$ if the above behavior is followed. We find linear behavior for large $\tau$ for $\alpha \leqslant 32$, with values for $p$ in the range $0.30-0.40$. (For $\alpha=64$ and 256 , the asymptotic region was evidently not reached.) Thus we conclude that the data is more consistent with the prediction of Talbot et al. than with Feder's law. Although the data in Fig. 1 appears to validate Feder's law, we note that only the last few points fall on the straight line. With values of $p$ in the range $0.30-0.40$, many more points follow a straight line in a similar plot.

The extrapolated value of $\theta_{j}(\alpha)$ depends upon $p$. However, by reanalyzing our $\theta(\tau)$ data with these new values of $p$, we find only a small change in the values of $\theta_{j}(\alpha)$ from those reported above and no change in the qualitative behavior. Specifically, we still find a maximum in $\theta_{j}(\alpha)$ at $\alpha \approx 2$, with $\theta_{j}(2)=0.551$ (rather than 0.545 ). For squares, $\theta_{j}(1)$ $\approx 0.532$ rather than 0.523 . In general, the values come out $\approx 0.01$ higher than those given in the text. The asymptotic behavior of $\theta_{j}(\alpha)$ for large $\alpha$ remains power-law as in Fig. 5 for $\theta_{j} \approx 0.70 \alpha^{-0.195}$.

We are now carrying out additional simulations in order to determine the long-time behavior more precisely and these results will be reported in a future paper. We thank Talbot et al. for sending us a copy of their work prior to its publication.

We acknowledge National Science Foundation Grant No. DMR-8619731 for support of this research. We thank H. S. Fogler and Ayumu Yokoyama for discussions which led to this work.

${ }^{1}$ Y. Pomeau, J. Phys. A 13, L193 (1980).

${ }^{2}$ Robert H. Swendsen, Phys. Rev. A 24, 504 (1981)

${ }^{3}$ Paul J. Flory, J. Am. Chem. Soc. 61, 1518 (1939).

${ }^{4}$ J. W. Evans, D. R. Burgess, and D. K. Hoffman, J. Chem. Phys. 79, 5011 (1983).

${ }^{5}$ Jens Feder, J. Theor. Biol. 87, 237 (1980).

${ }^{6} \mathrm{~L}$. Finegold and J. T. Donnell, Nature 278, 443 (1979).

${ }^{7}$ A. Renyi, Publ. Math. Inst. Hung. Acad. Sci. 3, 109 (1958).

${ }^{8}$ J. K. MacKenzie, J. Chem. Phys. 37, 723 (1962).

${ }^{9}$ B. Widom, J. Chem. Phys. 44, 3888 (1966).

${ }^{10}$ J. J. Gonzalez, P. C. Hemmer, and J. S. Høye, Chem. Phys. 3, 228 (1974).

${ }^{1}$ M. G. Kendall and P. A. P. Moran, Geometrical Probability (Hafner, New York, 1963).

${ }^{12}$ A. Palásti, Publ. Math. Inst. Hung. Acad. Sci. 5, 353 (1960).

${ }^{13}$ H. Solomon, Proc. 5th Berkeley Symp. Math. Stat, Probab. 3, 119 (1967).

${ }^{14}$ Y. Akeda and M. Hori, Nature 254, 318 (1975).

${ }^{15}$ H. J. Weiner, J. Appl. Prob. 15, 803 (1978).

${ }^{16}$ E. M. Tory and D. K. Pickard, J. Appl. Probab. 16, 697 (1979).

${ }^{17}$ L. A. Rosen, N. A. Seaton, and E. D. Glandt, J. Chem. Phys. 85, 7359 (1986).

${ }^{18}$ P. Meakin, John L. Cardy, E. Loh Jr., and D. J. Scalapino, J. Chem. Phys. 86, 2380 (1987).

${ }^{19}$ G. C. Barker and M. J. Grimson, Mol. Phys. 63, 145 (1988).

${ }^{20}$ P. Schaaf and J. Talbot, Phys. Rev. Lett. 62, 175 (1989).

${ }^{21}$ A. Yokoyama, K. R. Srinivasan, and H. S. Fogler, J. Colloid Interface Sci. 126, 141 (1988).

${ }^{22}$ J. Talbot, G. Tarjus, and P. Schaaf, Phys. Rev. A (in press). 